\title{
Uma abordagem baseada em redes neurais artificiais sobre o espectro de potência de eletroencefalogramas para o auxílio médico na classificação de crises epiléticas
}

\author{
Dionathan Luan de $\operatorname{Vargas}^{1}$, Jefferson Tales Oliva ${ }^{1}$, Marcelo Teixeira ${ }^{1}$ \\ ${ }^{1}$ Programa de Pós-Graduação em Engenharia Elétrica (PPGEE) \\ Universidade Tecnológica Federal do Paraná (UTFPR), Pato Branco - PR, Brasil \\ dionathan_vargas@hotmail.com, \{jeffersonoliva,mtex\}@utfpr.edu.br
}

\begin{abstract}
Epilepsy is the fourth most common neurological disorder in the world, affecting approximately 1\% of the population. Its diagnosis is supported by an electroencephalogram (EEG) that is further interpreted by a human specialist and may lead to divergent opinions, besides to be a tedious, inaccurate, and error-prone task. This paper proposes the automatic pattern recognition in the power spectrum of EEG segments, based on machine learning and feature engineering techniques. Results show the possibility of detecting epileptic crisis with accuracy greater than $80 \%$ in datasets used in the literature.
\end{abstract}

Resumo. A epilepsia é a quarta enfermidade neurológica mais comum e atinge aproximadamente $1 \%$ da população mundial. O diagnóstico é, em geral, amparado por um eletroencefalograma (EEG), cuja análise depende da interpretação médica, o que por vezes gera incongruência de diagnóstico, além de ser um trabalho tedioso, impreciso e propenso a erros. Este trabalho propõe um método de reconhecimento automático de padrões baseado em aprendizado de máquina e engenharia de características aplicadas ao espectros de potência de segmentos de EEGs. Resultados sugerem a possibilidade de detectar crises epilépticas com uma precisão superior a $80 \%$ em bases de dados já utilizadas na literatura.

\section{Introdução}

A epilepsia é um distúrbio neurológico definido por uma manifestação repetitiva de atividade elétrica excessiva de células nervosas. Cerca de 50 milhões de pessoas sofrem de epilepsia no mundo [OMS 2019], dos quais $80 \%$ vivem em países em desenvolvimento. No Brasil, a maioria dos estudos sobre a incidência desta doença são feitos apenas em grandes centros (e.g. [Gallucci Neto e Marchetti 2005] e [Häfele et al. 2018]), mas estima-se que, no geral, entre $0,4 \%$ e $1 \%$ da população pode ter este distúrbio [OMS 2019]. Essa alta taxa de incidência explicita a relevância para o desenvolvimento de tecnologias assistivas, direcionadas ao suporte de diagnóstico médico.

Para o diagnóstico de epilepsia, é comumente utilizado o eletroencefalograma (EEG) [Shin et al. 2014], que registra em séries temporais os impulsos elétricos cerebrais do paciente. A detecção de padrões relacionados a doenças em EEG é geralmente feita de forma visual por médicos especialistas. Além de demandar muito tempo, esse processo é também suscetível a erros humanos e a divergências de opiniões entre especialistas sobre uma mesma anormalidade evidenciada em EEGs [Oliva e Rosa 2019]. 
Uma alternativa para o suporte médico ao diagnóstico da epilepsia é dada por meio de sistemas de reconhecimento automático de padrões que, em segmentos de EEGs, consiste da extração de características do sinal para, em seguida, realizar a sua classificação. Neste contexto, métodos computacionais podem ser aplicados para a extração de características e aprendizado de máquina (AM) pode ser utilizado para a construção de classificadores a fim de automatizar o processo de detecção de crises epiléticas.

$\mathrm{Na}$ extração de características, duas formas são mais utilizadas na literatura: a extração por meio de aprendizado de características, que deixa a cargo do algoritmo decidir quais características são as mais importantes, como Convolutional Neural Network (CNN) [Wen e Zhang 2018, Gao et al. 2020]; e a engenharia de características, um processo manual, que consiste em computar medidas (e.g. média, desvio-padrão, entre outras) do conjunto de dados de entrada para o treinamento de classificadores [Bhattacharyya e Pachori 2017, Raghu et al. 2019].

Em segmentos de EEGs podem ser extraídas características temporais, espaciais e espectrais. A extração destas características é um tópico bastante fértil de pesquisas, já que as características afetam diretamente o desempenho dos classificadores [Cura e Akan 2021]. Entre os domínios espectrais, pode-se destacar o espectro de potência, que além de possuir um bom poder de descriminação para classificadores de EEG [Riaz et al. 2015], exige pouco poder computacional para gerá-lo, comparado ao espectrograma e biespectrograma.

Independente do domínio e do método de extração de características adotado, o processo de classificação de EEG é, em geral, conduzido da seguinte forma: inicialmente a base de dados é dividida em dados de treinamento e de teste. Ambos passam pelo mesmo processo de extração de características, mas as características dos dados de treinamento servem para treinar os classificadores, enquanto as dos dados de teste servem para estimar os classificadores. Estes identificam, então, a qual classe de EEG aquele sinal pertence, com base nas características extraídas. Quando referente a EEG de indivíduos com epilepsia, as classes podem pertencer aos estágios pré-ictais, ictais, pós-ictal e interictal [Fisher et al. 2014, Hwang et al. 2019].

Apesar de as redes neurais serem bastante utilizadas para solucionar problemas de classificação de EEG, a maioria dos trabalhos tem foco na diferenciação entre EEG normal e anormal. Alguns trabalhos, como [Nicolaou e Georgiou 2012] e [Mansour et al. 2020], têm um enfoque multi-classe, mas eles são a minoria, o que sugere que os modelos existentes podem ser eficientes para classificação de alguns estágios de sinais epiléticos, e ineficientes para outros.

Com base neste contexto, este trabalho explora os eventos epiléticos em segmentos de EEGs por meio de modelos preditivos, gerados a partir de métodos de aprendizado de máquina e engenharia de características aplicadas ao espectros de potência. Assim, os resultados permitem diferenciar sinais saudáveis de eventos pré-ictais e ictais, sendo um importante complemento às abordagens da literatura.

\section{Revisão da literatura}

Diversas técnicas de aprendizado de máquina têm sido utilizadas para detecção de epilepsia em segmentos de EEG representados por espectro de potência. Geralmente, este 
estudo é feito dividindo-se todo o espectro de potência em sub-brandas representativas como delta $(1-4 \mathrm{~Hz})$, teta $(4-8 \mathrm{~Hz})$, alfa $(8-12 \mathrm{~Hz})$, beta $(12-30 \mathrm{~Hz})$ e gama $(30-$ $60 \mathrm{~Hz}$ ) [Freeman e Quiroga 2012]. Esta sessão resume alguns trabalhos da última década encontrados na literatura.

Em [Nicolaou e Georgiou 2012] foi adotado uma abordagem baseada na extração de características por permutação de entropia. Como classificador foi utilizado Suport Vector Machines (SVM) e como validação, o algoritmo de Monte Carlo.

Em [Manjusha e Harikumar 2016], o espectro de potência foi utilizado como método de redução de dimensionalidade por poder lidar com dados complexos e não lineares. Por fim explorou classificadores através dos métodos KNN e k-means para EEG extraídos de vinte pacientes com epilepsia do hospital Sri Ramakrishna da Índia.

Novamente, o espectro de potência é objeto de estudo em [Tsipouras 2019], que investiga sub-bandas de frequência até então não exploradas na literatura. Para construção do classificador foi utilizado o método Randon Forest (RF) e, como entrada do algoritmo de AM, foram extraídas características baseadas em energia e entropia.

Além dos trabalhos supracitados, outras iniciativas focam em aprendizado profundo para a identificação da epilepsia, entretanto, elas não serão descritas aqui, pois essa seção se concentra em métodos que utilizam de engenharia de características aplicada ao espectro de potência de EEGs. Métodos de aprendizado profundo somente serão retomados na Sessão 4.1, para comparação de resultados.

Apesar da variedade de métodos apresentados na literatura a grande parte dos trabalhos é direcionado para classificação de apenas duas classes de sinais de EEG, como normal vs. interictal, normal vs. ictal, interictal vs. ictal e normal vs. ictal. Neste trabalho foi utilizada engenharia de características para a extração de 145 medidas de espectro de potência em segmentos de EEG para detecção de crises epiléticas por meio de redes neurais artificiais. Em geral, na literatura são extraídas poucas características dos sinais, e acredita-se que isso possa ser melhorado com a extração de um número maior de características de EEGs, facilitando assim a convergência dos algoritmos de aprendizado de máquina até para os casos mais complexos de classificação.

\section{Materiais e métodos}

\subsection{A base de dados}

Para a avaliação do modelo proposto neste artigo, foi utilizada a base de dados EEG Epileptogie Bonn [Andrzejak et al. 2001], disponível publica e gratuitamente ${ }^{1}$.

Esta base é utilizada em diversos estudos sobre detecção de crises epilépticas e é dividida em cinco subconjuntos definidos de A a E. Cada subconjunto contém 100 segmentos de EEG de um único canal com duração de 23,6 segundos. A taxa de amostragem do sinal é de 173,61 Hz e a resolução, de 12 bits. Amostras de segmentos de EEG podem ser vistos na Figura 1.

Os subconjuntos A e B são formados por dados de indivíduos saudáveis com os olhos abertos e fechados respectivamente. Os subconjuntos $\mathrm{C}$ e D estão relacionados à

\footnotetext{
${ }^{1}$ https://bit.ly/35qeHEm.
} 


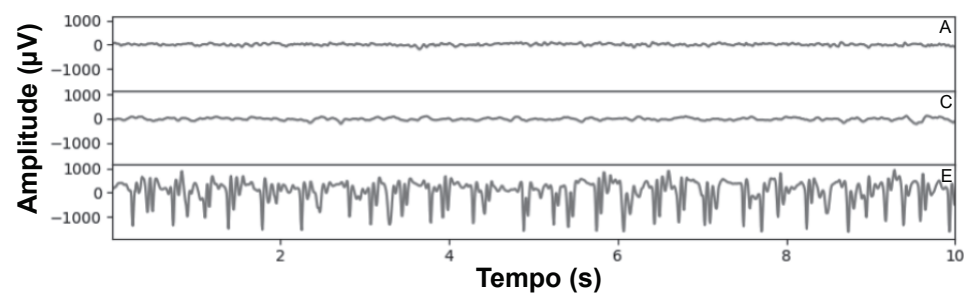

Figura 1. Exemplos de segmentos de EEG dos subconjuntos $A, C$ e $E$ da base de dados de Bonn.

fase interictal. O subconjunto $\mathrm{C}$ contem dados obtidos através da formação hipocampal do lado oposto do cérebro e D dados obtidos na zona epileptogênica (ZE). Por fim, o subconjunto E composto por dados ictais.

\subsection{Representação do sinal no domínio de frequência}

Sinais biológicos, como o EEG, são geralmente apresentados no domínio do tempo. No entanto, o sinal pode conter padrões que não sejam detectáveis neste domínio. Neste contexto, a análise do sinal no domínio de frequência pode ser mais informativa [Babadi e Brown 2014]. Para isso, primeiramente, os sinais no domínio do tempo devem ser convertidos por meio de algum método matemático. Neste trabalho foi adotado o método multitaper [Thomson 1982] que pode ser computado pela Equação 1, em que $h_{i, k}$ é o k-ésimo autovetor utilizado como uma função de janela (tapering), $X[k]$ é $k$-ésimo componente de frequência, $N$ é o comprimento do sinal, $j$ é a unidade imaginária da expressão complexa e $\omega$ é a frequência angular.

$$
X[k]=\sum_{i=1}^{N} h_{i, k} x[n] e^{-j \omega n} .
$$

Este método consiste na operação de correlação-cruzada entre um sinal representado no domínio do tempo e a equação de Euler [Babadi e Brown 2014] com a inclusão de k-tapers que são gerados por sequências slepianas [Slepian e Pollak 1961]. Estes tapers são ortogonais um do outro, por tanto, são gerados $k$ amostras de dados chamadas de subespectro. Essas estimativas espectrais ortogonais são então ponderadas, geralmente, usando pesos uniformes para obter o espectro médio denominado espectro multifuncional [Upadhya et al. 2018].

Em suma o espectro de potência (EP) é uma representação do espectro de frequência calculada por $P S(k)=|X[k]|^{2}$ [Kramer e Gerhardt 2012]. O espectro de potência de três amostras da base de Bonn podem ser vistas na Figura 2.

\subsection{Redes Neurais Artificiais}

As Redes Neurais Artificiais (RNA) compreendem técnicas computacionais inspiradas na estrutura neural biológica. Essas redes são amplamente utilizadas nas áreas das engenharias, por terem a habilidade de aprender a partir dos dados de entrada [Kalogirou 2001].

Pode-se dizer que uma RNA é uma representação matemática de elementos de computação interligados, conhecidos como neurônios artificiais, que podem estar divididos em três camadas: a camada de entrada, que é responsável por receber os dados na 


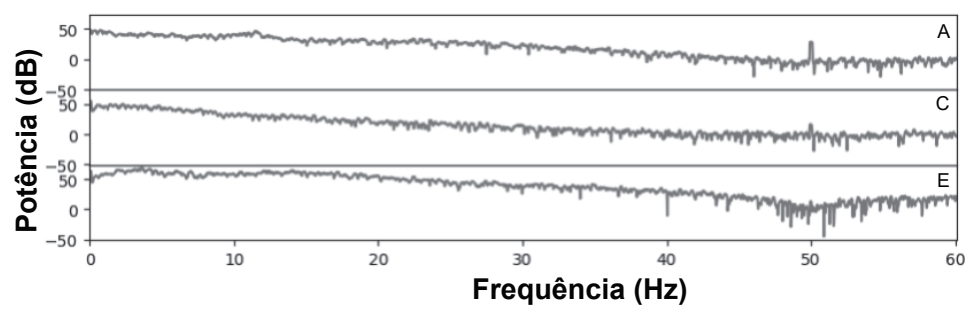

Figura 2. Exemplos de espectro de potência de segmentos de EEG dos subconjuntos $A, C$ e E da base de dados de Bonn.

entrada da RNA; as camadas ocultas, que são os neurônios responsáveis pelo processamento da informação de entrada através de conexões ponderadas; e a camada de saída, que fornecem os resultados do processamento das informações [Faceli et al. 2011].

Cada neurônio possui $n$ entradas referentes as saídas dos neurônios da camada anterior. Estas entradas são somadas de forma ponderada por pesos $w$ e combinados por uma função $f_{a}$, chamada de função de ativação. Várias funções podem ser utilizadas como função de ativação em um neurônio. Neste artigo iremos abordar 6 funções de ativação diferentes: tangente hiperbólica, sigmoide, softplus [Nair e Hinton ], Exponential Linear Unit (ELU) [Clevert et al. 2015], Scaled Exponential Linear Unit (SELU) [Klambauer et al. 2017] e Rectified Linear Unit (RELU) [Fukushima 1969]. A saída desta função é a resposta do neurônio para a entrada.

$\mathrm{O}$ treinamento comumente toma como base o algoritmo backpropagation [Rumelhart et al. 1986], que se divide em duas etapas, na primeira etapa denominada forward, onde o conjunto de treinamento é utilizado como entrada da rede e propagado camada a camada até a produção das saídas, as quais são comparadas com as saídas verdadeiras, cuja diferença é chamada de erro. Em uma segunda etapa denominada backward, o erro é retro-propagado pela rede ajustando os pesos para as classes verdadeiras dos exemplos de treinamento. Em suma, as sucessivas aplicações das fases forward e backward fazem com que os pesos se ajustem a cada interação, implicando na diminuição dos erros e fazendo com que a rede aprenda com os dados amostrados. Estes ciclos são repetidos até que se atinja um critério de parada que pode ser um número máximo de ciclos ou um valor especifico em uma métrica avaliada [Faceli et al. 2011].

\subsection{Configuração experimental}

Neste trabalho, os classificadores RNA foram construídos utilizando a linguagem $P y$ thon $^{2}$ com o auxilio das bibliotecas Tensorflow ${ }^{3}$ e scikit-learn ${ }^{4}$ por facilitarem a implementação algoritmos de AM.

Os modelos foram construídos em três etapas: geração do EP, extração de características e construção de classificadores. Na primeira etapa, foi gerado o EP, através do método multitaper, para cada segmento de EEG.

Na extração de características, o sinal foi decomposto em bandas de frequência: delta, teta, alfa, beta e gama. Para cada banda foram computadas 29 medidas, ge-

\footnotetext{
${ }^{2}$ https://www.python.org/

${ }^{3}$ https://www.tensorflow.org/

${ }^{4}$ https://scikit-learn.org/
} 
rando um total de 145 características. Estas características passaram por um processo de normalização pela equação $z=(x-u) / s$, onde $x$ é o vetor de características, $u$ é a média do vetor e $s$ o desvio padrão. Desta forma, nenhum parâmetro terá uma maior influência na rede devido a sua amplitude. As características que foram extraídas de cada sub-banda são apresentadas na Tabela 1 .

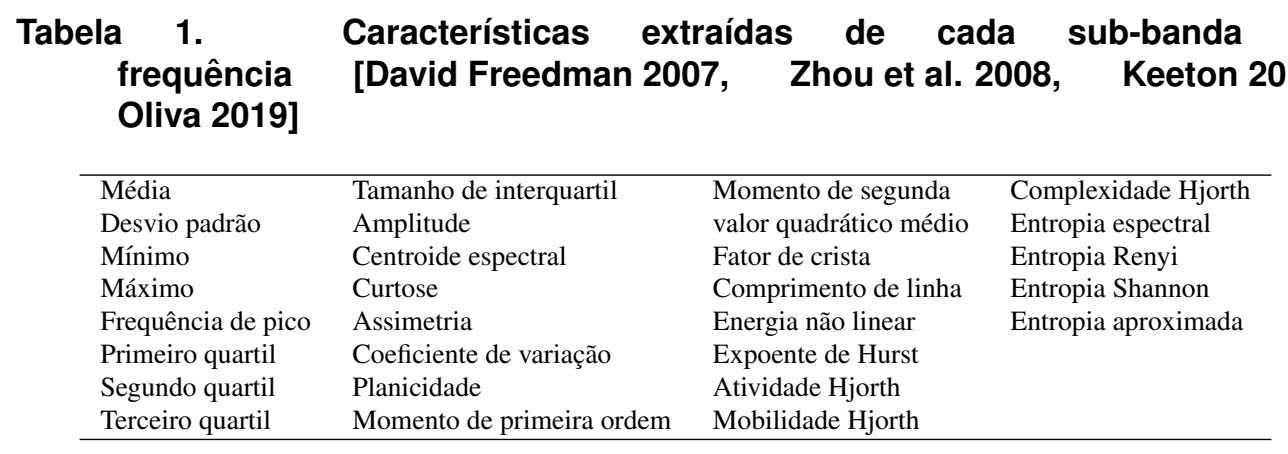

$\mathrm{Na}$ etapa de construção de classificadores, algumas arquiteturas perceptron multicamadas totalmente conectadas foram avaliadas para o caso mais complexo da base de Bonn, a classificação de cinco classes. Todas as redes tinham em comum: a camada de entrada composta por 145 neurônios referente às características extraídas do EP; e a camada de saída, composta por cinco neurônios com a ativação softmax que retorna um vetor de probabilidade. Neste caso é a probabilidade do segmento de EEG da entrada da rede ser referente a uma das cinco classes consideradas neste estudo.

Como uma RNA com apenas uma camada oculta pode implementar qualquer função contínua para classificação [Cybenko 1989], apenas foi variada a quantidade de neurônios e a função de ativação da camada oculta a fim de atingir o maior desempenho. Sendo assim, foram testadas redes com 64, 128, 256, 512, 1024, 2048 e 4096 neurônios, considerando funções de ativação, tais como tangente hiperbólica, sigmóide, softplus, ELU, SELU e RELU.

Cada modelo foi treinado utilizando a estratégia de incrementar a quantidade de épocas até atingir a acurácia máxima nos dados de treino. Isso ocorreu por volta de 500 épocas utilizando o algoritmo back-propagation, e por isso, esta quantidade de épocas foi utilizada para os demais casos. Como métrica de desempenho, nesta etapa, foi utilizada apenas a acurácia, gerada na aplicação da validação-cruzada com 10 folds de cada modelo.

O modelo com maior desempenho ainda foi testado em outros 6 casos da base de Bonn explorados na literatura, sendo cinco casos de duas classes e um caso com três classes. A lista dos casos de estudo são apresentados na Tabela 2.

Tabela 2. Casos de teste.

\begin{tabular}{lllr}
\hline & Conjuntos & Descrição & Número de amostras \\
\hline Caso 1 & A vs. E & Saudável (olhos abertos) vs. Ictal & 200 \\
Caso 2 & B vs. E & Saudável (olhos fechados) vs. Ictal & 200 \\
Caso 3 & C vs. E & Interictal oposta a ZE vs. Ictal & 200 \\
Caso 4 & D vs. E & Interictal na ZE vs. Ictal & 200 \\
Caso 5 & ABCD vs. E & Sem crise vs. Com crise & 500 \\
Caso 6 & AB vs. CD vs. E & Saudável vs. Interictal vs. Ictal & 500 \\
Caso 7 & A vs. B vs. C vs. D vs. E & Todas as classes & 500 \\
\hline
\end{tabular}




\subsection{Métricas de avaliação}

Para mensurar os resultados obtidos, foram usadas métricas clássicas da literatura, como Acurácia (ACU), Precisão (PRE), Especificidade (ESP) e Sensitividade (SEN). A métrica F1 [Lewis e Gale 1994] vem sendo abordada pela maioria dos sistemas modernos de classificação de epilepsia e, por isso, também será considerada neste artigo. Estas métricas darão suporte para a comparação com outros trabalhos e são definidas a seguir, tendo VP como os verdadeiros positivos, FN são os falsos negativos, VN os verdadeiros negativos, FP os falsos positivos e $\mathrm{N}$ a quantidade total de amostras testadas.

$$
\begin{gathered}
\mathrm{ACU}=\frac{\mathrm{VP}}{\mathrm{N}} ; \\
\mathrm{SEN}=\frac{\mathrm{VP}}{\mathrm{FN}+\mathrm{VP}} ;
\end{gathered}
$$

$$
\mathrm{PRE}=\frac{\mathrm{VP}}{\mathrm{VP}+\mathrm{FP}}
$$

$$
\begin{gathered}
\mathrm{ESP}=\frac{\mathrm{VN}}{\mathrm{VN}+\mathrm{FP}} ; \\
F 1=\frac{2 * \mathrm{PRE} * \mathrm{SEN}}{\mathrm{PRE}+\mathrm{SEN}} .
\end{gathered}
$$

\section{Resultados e Discussões}

Para seleção do melhor modelo foram testados diferentes funções de ativação e quantidades de neurônios na camada intermediária para o Caso 7 como proposto na Sessão 3.4. O resultado do teste de seleção de modelo pode ser visto na Figura 3. Apesar do aumento da acurácia nos testes, conforme os incrementos da quantidade de neurônios da camada intermediária, ficou evidente que as funções de ativação tangente hiperbólica e sigmoide foram menos eficiente comparadas às demais.

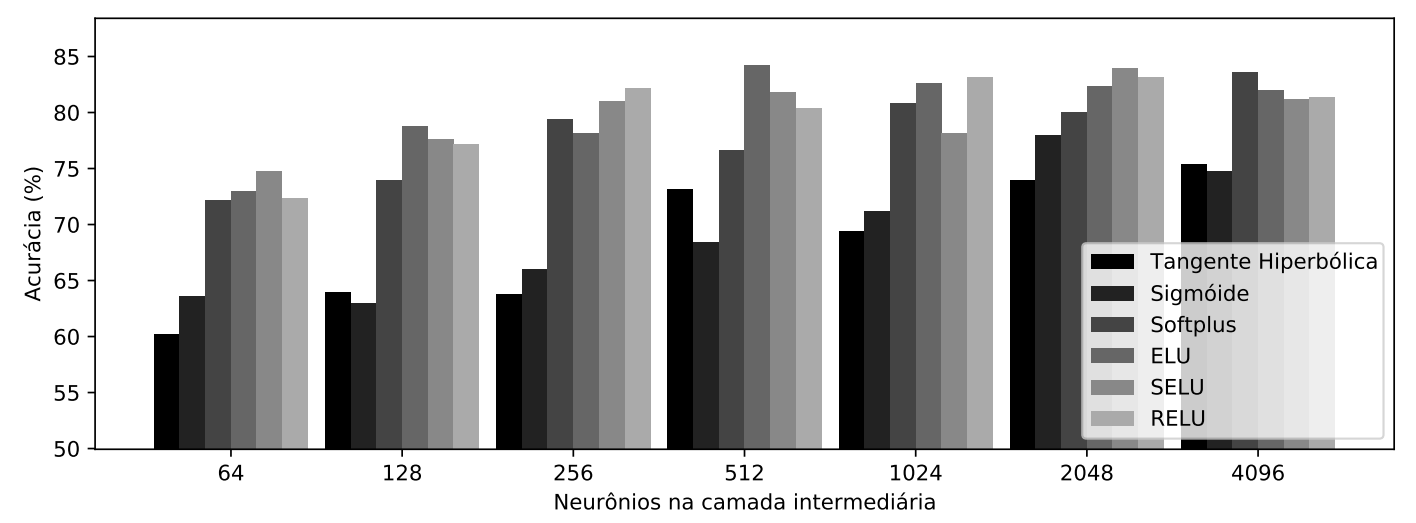

Figura 3. Acurácia das redes em função do número de neurônios na camada intermediária e diferentes funções de ativação

As redes com função de ativação softplus obtiveram o seu maior desempenho com 4096 neurônios, atingindo uma acurácia média de 83,6\% e um desvio padrão de 4,18\%. As redes com função de ativação RELU obtiveram acurácia de 83,2\% com 1024 e 2048 neurônios na camada intermediária, com desvio padrão de $4,58 \%$ e $6,54 \%$, respectivamente. As redes com função de ativação SELU obtiveram acurácia de $84 \%$ e um desvio padrão de 5,44\% com 2048 neurônios, ficando muito próxima do maior resultado, que foi das redes com função de ativação ELU, que obteve 84,2\% e um desvio padrão de 5,4\%, com 512 neurônios na camada intermediária.

De acordo com os resultados preliminares, pode-se dizer que o modelo com a camada intermediária de 512 neurônios e função de ativação ELU obteve uma melhor 
acurácia na aplicação validação-cruzada. Portanto, para os próximos experimentos apresentados este foi o modelo adotado como base. Uma abordagem gráfica do método proposto pode ser visto Figura 4 tendo como única mudança a camada de saída que, dependendo do estudo de caso, terá sua quantidade de neurônios alterada de acordo com a quantidade de classes a serem avaliadas.

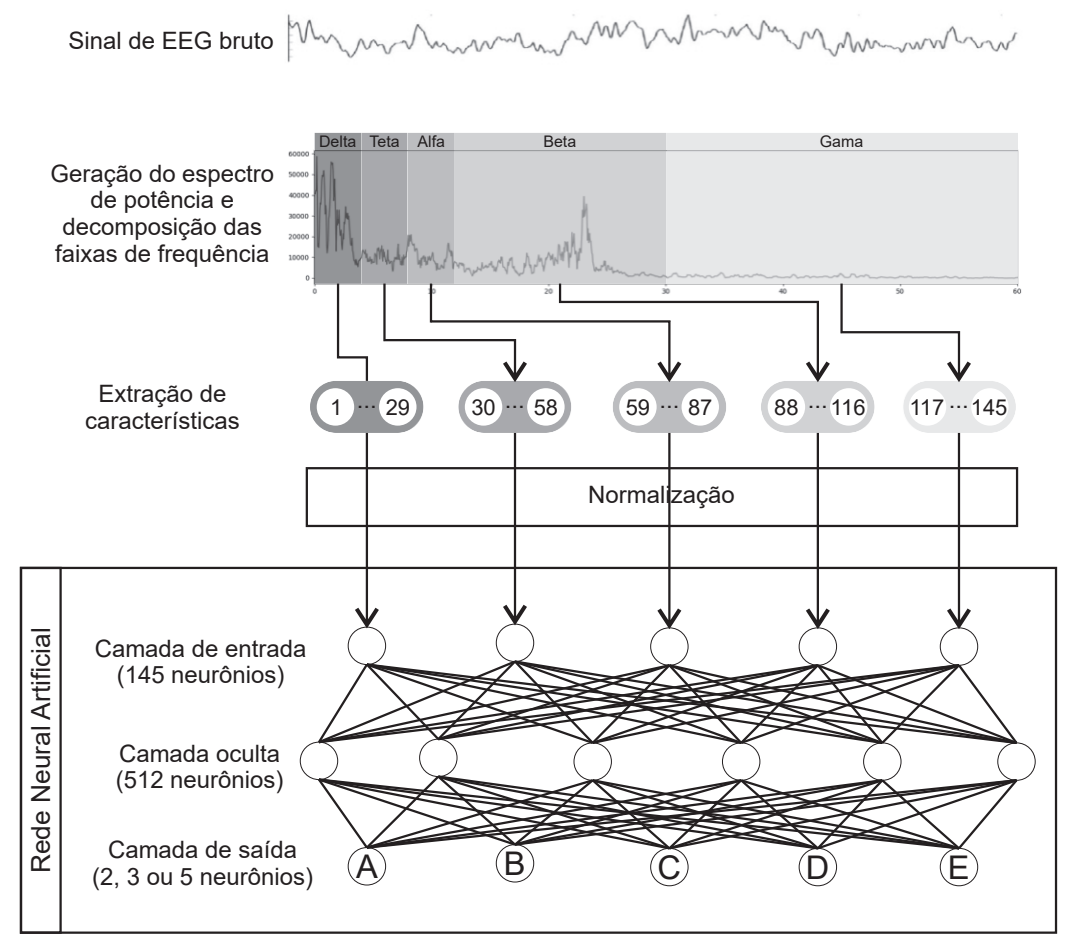

Figura 4. Fluxo de atividades que representa o método proposto.

\subsection{Resultados experimentais}

Para cada um dos casos da Tabela 2 foi gerado um modelo com 145 neurônios na camada de entrada, 512 neurônios com ativação ELU na camada oculta e camada de saída com neurônios com ativação softmax e quantidade igual a dos conjuntos a serem avaliadas. A média e o desvio padrão das medidas preditivas apresentadas na Seção 3.5, referente a estes modelos são apresentados na Tabela 3.

Tabela 3. Resultados em percentual (\%) dos estudos de caso.

\begin{tabular}{lccccccc}
\hline Métrica & Caso 1 & Caso 2 & Caso 3 & Caso 4 & Caso 5 & Caso 6 & Caso 7 \\
\hline ACU & $100,0 \pm 0,00$ & $100,0 \pm 0,00$ & $100,0 \pm 0,00$ & $99,50 \pm 1,50$ & $98,60 \pm 1,80$ & $97,40 \pm 1,80$ & $84,20 \pm 5,40$ \\
PRE & $100,0 \pm 0,00$ & $100,0 \pm 0,00$ & $100,0 \pm 0,00$ & $99,40 \pm 1,67$ & $97,64 \pm 3,19$ & $97,60 \pm 1,70$ & $86,00 \pm 4,30$ \\
SEN & $100,0 \pm 0,00$ & $100,0 \pm 0,00$ & $100,0 \pm 0,00$ & $99,58 \pm 1,25$ & $98,50 \pm 2,21$ & $97,24 \pm 2,18$ & $84,45 \pm 5,51$ \\
ESP & $100,0 \pm 0,00$ & $100,0 \pm 0,00$ & $100,0 \pm 0,00$ & $99,50 \pm 1,50$ & $98,60 \pm 1,80$ & $98,70 \pm 0,90$ & $96,05 \pm 1,35$ \\
F1 & $100,0 \pm 0,00$ & $100,0 \pm 0,00$ & $100,0 \pm 0,00$ & $99,51 \pm 1,46$ & $98,55 \pm 1,96$ & $97,42 \pm 1,88$ & $85,20 \pm 4,82$ \\
\hline
\end{tabular}

Os modelos dos casos A vs. E, B vs. E e C vs. E atingiram valores máximos para as medidas de desempenho. No teste $\mathrm{D} v s$. E, o modelo obteve uma acurácia próxima à máxima com 99,5\% de acerto. Pode-se notar, também, que neste último caso o desvio padrão das métricas se mostrou baixo (menor que 2\%), reforçando a integridade da rede utilizada. Para o caso ABCD vs. E, a acurácia média foi de 98,6\%, superando alguns modelos propostos na literatura. 
Como esperado, devido à maior complexidade, os casos multiclasse estudados apresentaram desempenho inferior aos casos de duas classes. No caso AB vs. CD vs. E obteve-se uma acurácia de 97,40\%, maior em relação a alguns trabalhos já publicados. Para o caso A vs. B vs. C vs. D vs. E, o modelo atingiu uma acurácia de 84,20\%.

$\mathrm{Na}$ Tabela 4 é apresentada uma comparação dos nossos resultados com alguns trabalhos relacionados aplicados à base de Bonn ${ }^{5}$. Nos estudos de caso 1, 2, 3 e 4, o sistema proposto atingiu o mesmo desempenho dos trabalhos do estado da arte. Nos casos 5,6 , e 7, o trabalho [Li et al. 2020] possui a maior acurácia encontrada na literatura. Tal desempenho foi obtido integrando uma análise espectral multinível e a análise temporal multiescalar, simultaneamente integrado a uma CNN para extração de características. Por fim, a rede de classificação é implementada através de RNA para reconhecimento de EEG epiléptico com base em recursos extraídos pela CNN.

Tabela 4. Comparação dos resultados com outros trabalhos aplicados a base de Bonn.

\begin{tabular}{llcllc}
\hline Caso & Trabalho & Acurácia & Caso & Trabalho & Acurácia \\
\hline A vs E & [Sharmila e Geethanjali 2016] & $100,0 \%$ & ABCD vs E & [Riaz et al. 2015] & $96,00 \%$ \\
& [Tzallas et al. 2012] & $100,0 \%$ & & [Tzallas et al. 2012] & $97,70 \%$ \\
& Este trabalho & $\mathbf{1 0 0 , 0 \%}$ & & [Sharma et al. 2017] & $99,20 \%$ \\
B vs E & [Siuly et al. 2018] & $99,00 \%$ & & [Li et al. 2020] & $\mathbf{9 9 , 8 0 \%}$ \\
& [Sharma et al. 2017] & $100,0 \%$ & & Este trabalho & $98,60 \%$ \\
& Este trabalho & $\mathbf{1 0 0 , 0 \%}$ & AB vs CD vs E & [Riaz et al. 2015] & $83,00 \%$ \\
C vs E & [Siuly et al. 2018] & $98,50 \%$ & & [Zhang e Chen 2016] & $98,40 \%$ \\
& [Sharma et al. 2017] & $99,00 \%$ & & [Li et al. 2020] & $\mathbf{9 9 , 3 6 \%}$ \\
& [Aydemir et al. 2020] & $100,0 \%$ & & Este trabalho & $97,40 \%$ \\
& Este trabalho & $\mathbf{1 0 0 , 0 \%}$ & A vs B vs C vs D vs E & [Nicolaou e Georgiou 2012] & $86,10 \%$ \\
D vs E & [Sharma et al. 2017] & $98,50 \%$ & & [Tsipouras 2019] & $91,20 \%$ \\
& [Aydemir et al. 2020] & $99,50 \%$ & & [Li et al. 2020] & $\mathbf{9 4 , 6 0 \%}$ \\
& Este trabalho & $\mathbf{9 9 , 5 0 \%}$ & Este trabalho & $84,20 \%$ \\
\hline
\end{tabular}

\section{Conclusão}

Este artigo apresentou um método de reconhecimento inteligente de EEGs por meio de RNAs e de engenharia de características aplicadas ao EP para detecção automática de eventos epileptiformes. O desempenho de classificação da abordagem proposta foi avaliado em um conjunto de dados disponível publica e gratuitamente. Tal conjunto é composto por cinco classes, formadas por segmentos de EEG coletados de pessoas sem epilepsia, e por segmentos de sinais coletados no momento da crise.

Os resultados experimentais sugerem que a abordagem proposta resulta em um nível de acurácia igual ou superior aos trabalhos da literatura, na maioria dos casos. Apenas no caso mais complexo, i.e., na separação das cinco classes da base de Bonn, o método proposto obteve uma acurácia menor em comparação com alguns trabalhos relacionados. Ainda assim, entende-se que eles sejam promissores, uma vez que não há registros de modelos que, implementando a engenharia de características de EP e RNA, resultem em melhor acurácia, para este caso na base de Bonn. Assim, nossas perspectivas de trabalhos futuros se pautam na exploração das características aqui apresentadas, visando reduzir a complexidade da rede e melhorar o seu desempenho.

\footnotetext{
${ }^{5} \mathrm{Nem}$ todos os trabalhos citados são baseados em espectro de potência e foram selecionados pelo desempenho obtido nos testes.
} 


\section{Agradecimentos}

O presente trabalho foi realizado com apoio da Coordenação de Aperfeiçoamento de Pessoal de Nível Superior - Brasil (CAPES) - Código de Financiamento 001, do Conselho Nacional de Desenvolvimento Científico e Tecnológico (CNPq), da Fundação Araucária (FA) e da Financiadora de Estudos e Projetos (FINEP).

\section{Referências}

Andrzejak, R. G., Lehnertz, K., Mormann, F., Rieke, C., David, P., and Elger, C. E. (2001). Indications of nonlinear deterministic and finite-dimensional structures in time series of brain electrical activity: Dependence on recording region and brain state. Physical Review E, 64(6):061907.

Aydemir, E., Tuncer, T., and Dogan, S. (2020). A tunable-Q wavelet transform and quadruple symmetric pattern based EEG signal classification method. Medical hypotheses, 134:109519.

Babadi, B. and Brown, E. N. (2014). A review of multitaper spectral analysis. IEEE Transactions on Biomedical Engineering, 61(5):1555-1564.

Bhattacharyya, A. and Pachori, R. B. (2017). A multivariate approach for patient-specific EEG seizure detection using empirical wavelet transform. IEEE Transactions on Biomedical Engineering, 64(9):2003-2015.

Clevert, D.-A., Unterthiner, T., and Hochreiter, S. (2015). Fast and accurate deep network learning by exponential linear units (elus). arXiv preprint arXiv:1511.07289.

Cura, O. K. and Akan, A. (2021). Analysis of epileptic EEG signals by using dynamic mode decomposition and spectrum. Biocybernetics and Biomedical Engineering, 41(1):28-44.

Cybenko, G. (1989). Approximation by superpositions of a sigmoidal function. Mathematics of control, signals and systems, 2(4):303-314.

David Freedman, Robert Pisani, R. P. (2007). Statistics. W. W. Norton Co, 4th edition.

Faceli, K., Lorena, A. C., Gama, J., Carvalho, A., et al. (2011). Inteligência artificial: Uma abordagem de aprendizado de máquina. Rio de Janeiro: LTC, 2:192.

Fisher, R. S., Scharfman, H. E., and DeCurtis, M. (2014). How can we identify ictal and interictal abnormal activity? Issues in Clinical Epileptology: A View from the Bench, pages 3-23.

Freeman, W. and Quiroga, R. Q. (2012). Imaging brain function with EEG: advanced temporal and spatial analysis of electroencephalographic signals. Springer Science \& Business Media.

Fukushima, K. (1969). Visual feature extraction by a multilayered network of analog threshold elements. IEEE Transactions on Systems Science and Cybernetics, 5(4):322333.

Gallucci Neto, J. and Marchetti, R. L. (2005). Aspectos epidemiológicos e relevância dos transtornos mentais associados à epilepsia. Brazilian Journal of Psychiatry, 27(4):323328. 
Gao, X., Yan, X., Gao, P., Gao, X., and Zhang, S. (2020). Automatic detection of epileptic seizure based on approximate entropy, recurrence quantification analysis and convolutional neural networks. Artificial intelligence in medicine, 102:101711.

Häfele, C. A., Freitas, M. P., Gervini, B. L., de Carvalho, R. M., and Rombaldi, A. J. (2018). Who are the individuals diagnosed with epilepsy using the public health system in the city of pelotas, southern brazil? Epilepsy \& Behavior, 78:84-90.

Hwang, S. T., Goodman, T., and Stevens, S. J. (2019). Painful seizures: a review of epileptic ictal pain. Current pain and headache reports, 23(11):1-7.

Kalogirou, S. A. (2001). Artificial neural networks in renewable energy systems applications: a review. Renewable and sustainable energy reviews, 5(4):373-401.

Keeton, G. (2015). What is crest factor and why is it important? https : / / shorturl. at/yB23 4, Abril.

Klambauer, G., Unterthiner, T., Mayr, A., and Hochreiter, S. (2017). Self-normalizing neural networks. arXiv preprint arXiv:1706.02515.

Kramer, C. and Gerhardt, H. J. (2012). Advances in wind engineering. Elsevier.

Lewis, D. and Gale, W. (1994). Training text classifiers by uncertainty sampling. In seventeenth annual international ACM SIGIR conference on research and development in information retrieval, pages 3-12.

Li, Y., Liu, Y., Cui, W.-G., Guo, Y.-Z., Huang, H., and Hu, Z.-Y. (2020). Epileptic seizure detection in EEG signals using a unified temporal-spectral squeeze-andexcitation network. IEEE Transactions on Neural Systems and Rehabilitation Engineering, 28(4):782-794.

Manjusha, M. and Harikumar, R. (2016). Performance analysis of KNN classifier and Kmeans clustering for robust classification of epilepsy from EEG signals. In 2016 International Conference on Wireless Communications, Signal Processing and Networking (WiSPNET), pages 2412-2416. IEEE.

Mansour, A. M., Obeidat, M. A., and Al-Aqtash, M. (2020). Data mining based approach for evaluation of EEG signals for epilepsy detection. WSEAS Transactions on Biology and Biomedicine.

Nair, V. and Hinton, G. E. Rectified linear units improve restricted boltzmann machines. In Icml. https: / / redirect. is / xg939zy, Abril.

Nicolaou, N. and Georgiou, J. (2012). Detection of epileptic electroencephalogram based on permutation entropy and support vector machines. Expert Systems with Applications, 39(1):202-209.

Oliva, J. T. (2019). Geração automática de laudos médicos para o diagnóstico de epilepsia por meio do processamento de eletroencefalogramas utilizando aprendizado de máquina. $\mathrm{PhD}$ thesis, Universidade de São Paulo.

Oliva, J. T. and Rosa, J. L. G. (2019). Classification for EEG report generation and epilepsy detection. Neurocomputing, 335:81-95.

OMS (2019). Epilepsy: a public health imperative. https : / / shorturl . at / yKNQ6, Março. 
Raghu, S., Sriraam, N., Hegde, A. S., and Kubben, P. L. (2019). A novel approach for classification of epileptic seizures using matrix determinant. Expert Systems with Applications, 127:323-341.

Riaz, F., Hassan, A., Rehman, S., Niazi, I. K., and Dremstrup, K. (2015). Emd-based temporal and spectral features for the classification of EEG signals using supervised learning. IEEE Transactions on Neural Systems and Rehabilitation Engineering, 24(1):28-35.

Rumelhart, D. E., Hinton, G. E., and Williams, R. J. (1986). Learning representations by back-propagating errors. nature, 323(6088):533-536.

Sharma, M., Pachori, R. B., and Acharya, U. R. (2017). A new approach to characterize epileptic seizures using analytic time-frequency flexible wavelet transform and fractal dimension. Pattern Recognition Letters, 94:172-179.

Sharmila, A. and Geethanjali, P. (2016). DWT based detection of epileptic seizure from EEG signals using naive bayes and kNN classifiers. Ieee Access, 4:7716-7727.

Shin, H. W., Jewells, V., Hadar, E., Fisher, T., and Hinn, A. (2014). Review of epilepsyetiology, diagnostic evaluation and treatment. Int J Neurorehabilitation, 1(130):23760281 .

Siuly, S., Alcin, O. F., Bajaj, V., Sengur, A., and Zhang, Y. (2018). Exploring hermite transformation in brain signal analysis for the detection of epileptic seizure. IET Science, Measurement \& Technology, 13(1):35-41.

Slepian, D. and Pollak, H. O. (1961). Prolate spheroidal wave functions, Fourier analysis and uncertainty-I. Bell System Technical Journal, 40(1):43-63.

Thomson, D. J. (1982). Spectrum estimation and harmonic analysis. Proceedings of the IEEE, 70(9):1055-1096.

Tsipouras, M. G. (2019). Spectral information of EEG signals with respect to epilepsy classification. EURASIP Journal on Advances in Signal Processing, 2019(1):1-17.

Tzallas, A. T., Tsipouras, M. G., Tsalikakis, D. G., Karvounis, E. C., Astrakas, L., Konitsiotis, S., and Tzaphlidou, M. (2012). Automated epileptic seizure detection methods: a review study. Epilepsy-histological, electroencephalographic and psychological aspects, pages 75-98.

Upadhya, S. S., Cheeran, A., and Nirmal, J. H. (2018). Thomson multitaper MFCC and PLP voice features for early detection of parkinson disease. Biomedical Signal Processing and Control, 46:293-301.

Wen, T. and Zhang, Z. (2018). Deep convolution neural network and autoencoders-based unsupervised feature learning of EEG signals. IEEE Access, 6:25399-25410.

Zhang, T. and Chen, W. (2016). LMD based features for the automatic seizure detection of EEG signals using SVM. IEEE Transactions on Neural Systems and Rehabilitation Engineering, 25(8):1100-1108.

Zhou, S.-M., Gan, J. Q., and Sepulveda, F. (2008). Classifying mental tasks based on features of higher-order statistics from EEG signals in brain-computer interface. Information Sciences, 178(6):1629-1640. 